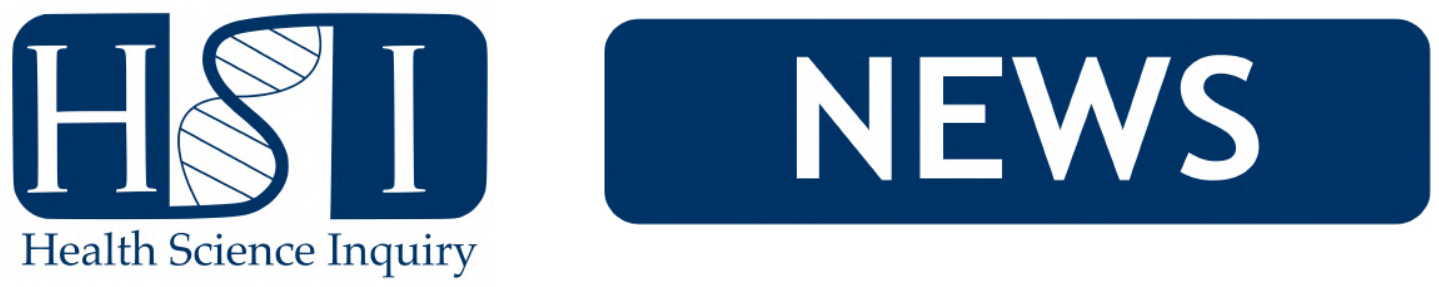

\title{
How Will Science Help to Address the Opioid Crisis?
}

\section{Lola Welsch}

You have probably heard about the opioid crisis: an epidemic of opioid abuse and overdoses of highly potent painkillers such as morphine, heroin or oxycodone - raging in North America since 2010. The number of opioid-related deaths surpassed 4,000 in 2017 in Canada alone, placing opioid use as the leading cause of premature deaths (above car accidents). On top of that, opioid abuse during pregnancy has led to a dramatic increase in drug-dependent newborns and has additionally contributed to the spread of infectious diseases such as HIV and hepatitis C [1]. If we take a look back, we can better understand the origins of this crisis: morphine, the active substance in opium extracted from poppies, has been used for centuries due to its unparalleled pain-relieving and euphoria-inducing properties. In the 1990s, the modern pharmacopeia introduced synthetic molecules such as oxycodone, marketed by pharmaceutical companies as safe, non-addictive pain-relievers. Subsequently, the prescription rate of opioids increased dramatically, reaching a peak in 2010, exposing a large numbers of North Americans to highly addictive drugs. Prescription opioids have been a gateway to non-medical use of opioids for many people: approximately $10 \%$ of patients with opioid prescription develop an opioid use disorder [2]. In addition to facilitating drug use among non-users, prescription opioids were also the main supply for the illicit market, making opioids available on the streets. In 2012, authorities grew increasingly recognizant of the harms resulting from over-prescription and took measures to reduce the prescription of opioids [1]. Despite these efforts, the crisis worsened with the introduction of a new, incredibly potent and easily synthesized opioid on the illicit market. Prescription-reliant opioid users, at times, had no choice but to turn to this hazardous drug, exacerbating the current opioid crisis. Several national measures have since been implemented to contain this global health issue: reduction of opioid prescription, intensification of the monitoring of prescribed opioids, creation of safe consumption centers and distribution of naloxone, an overdose-reversing drug. Despite these efforts, considerable work remains to overcome the crisis, and research has an important role to play [3].

The National Institute of Health (NIH) have drawn two lines of research to foster the efforts to address the epidemic: the first objective is reactive and focuses on improving care for opioid use and addiction. The second objective is proactive and attempts to mitigate opioid use by offering alternative pain relief techniques (Initiatives Research plans, NIH, 2018). In April 2018, the NIH started the HEAL program (Helping to End Addiction Long-term). The initiative encourages improvements to opioid use disorder therapies, in particular though the combination of psycho-therapies and medication-assisted treatment (promoting transition drugs such as buprenorphine or methadone).

Interestingly, computer-based tools are under development to broaden and facilitate access to psychiatric care, often in limited availability. For instance, the National Institute of Drug Abuse is developing an interactive program intended for cognitive-behavioral therapy (CBT). In essence, CBT is a form of psycho-therapy focused on treating specific maladaptive behaviors, problems, and symptoms. CBT has shown efficacy in addressing substance use disorders and coping with the disease. In this program, Dr. Caroll and colleagues use a combination of games, cartoons and interactive exercises to teach patients how to deal with stressful or risky situations without returning to drug use. These kinds of computerassisted therapies offer several advantages compared to currently available therapies; CBT could be the first step for patients to engage in a complete therapeutic process and to enhance the recovery rate. Their use also liberates time for the psycho-medical staff to manage in-person clinical therapies [4]. Other teams in the field of addiction therapy are working on tools for early diagnosis of substance use disorders. These include using innovative approaches such as stateof-the-art neuro-imaging techniques [5], or combining social media data with deep neural networks to identify at-risk populations [6]. All of these efforts are made in the hope to better address addiction disorders, parallel to traditional addiction prevention strategies.

The majority of opioids abusers are first introduced to these drugs via prescriptions for severe and/or chronic pain; despite the harmful and addictive potential of opioids, they remain the best class of drugs for pain relief. In this context, researchers are looking for opioid-like painkillers with fewer unwanted side-effects. To this end, some drug discovery strategies comprise designing "better opioids" by tweaking the cellular target of the drugs. Scientists have been able to manipulate the effects of the chemicals. Progress has been made in this field particularly with drugs called "biased opioids": as traditional opioids, they target the mu opioid receptor, responsible for all the effects of morphine. However, unlike traditional opioids, they are biased toward a specific cell signaling pathway, the one thought to be responsible 
for pain relief. It is also believed that they lessen the activation of the pathway thought to be responsible for tolerance development, respiratory depression, nausea and constipation. Other similar approaches to designing the ideal opioid involve identifying molecules acting at different opioid receptors. However, the question of the addictive potential of these drugs and their rewarding properties is still debated and likely will not be available in the clinic for many years [7]. To avoid opioids altogether, a considerable effort is taking place to develop new non-opioid analgesics. For instance, there is increasing evidence supporting the use of medical marijuana in treating chronic pain [8]. In parallel, other non-pharmacological approaches are underway: pilot studies with demonstrated efficacy of physiotherapy or meditation in the reduction of chronic pain offer some hope [9].

Science has taken a very diverse approach and tackles the opioid crisis from complimentary perspectives. Even if we can distinguish major priorities in research, this article is not an exhaustive list of all existing attempts to address the issue, but every idea and effort is an important piece of the puzzle. Importantly, the solution can only be complete by combining research, medicine, technologies, personal psychiatric care, public health interventions and harm reduction policies. To conclude, we can pinpoint that researchers and clinicians working in the field of opioid use disorders recommend talking about it and treating it as a chronic illness and not a moral failing. The first step in addressing the crisis would then be to go over the stigmatisation and marginalization of opioid use to make a change in how we choose to move for better care [10].

\section{REFERENCES}

[1] Fischer B, Pang M, Tyndall M. The opioid death crisis in Canada: crucial lessons for public health. The Lancet Public Health. 2019;4(2):e81-e82.

[2] Carlson RG, Nahhas RW, Martins SS, Daniulaityte R. Predictors of transition to heroin use among initially non-opioid dependent illicit pharmaceutical opioid users: A natural history study. Drug and alcohol dependence. 2016;160:127-134.

[3] Fischer B, Rehm J, Tyndall M. Effective Canadian policy to reduce harms from prescription opioids: learning from past failures. CMAJ. 2016;188(17-18):1240-1244.

[4] Carroll KM, Kiluk BD, Nich C, Gordon MA, Portnoy GA, Marino DR, et al. Computer-assisted delivery of cognitive-behavioral therapy: efficacy and durability of CBT4CBT among cocaine-dependent individuals maintained on methadone. American journal of Psychiatry. 2014;171(4):436-444.

[5] Moningka H, Lichenstein S, Worhunsky PD, DeVito EE, Scheinost D, Yip SW. Can neuroimaging help combat the opioid epidemic? A systematic review of clinical and pharmacological challenge fMRI studies with recommendations for future research. Neuropsychopharmacology. 2018;p. 1.

[6] Hassanpour S, Tomita N, DeLise T, Crosier B, Marsch LA. Identifying substance use risk based on deep neural networks and Instagram social media data. Neuropsychopharmacology. 2019;44(3):487.

[7] Severino AL, Shadfar A, Hakimian JK, Crane O, Singh G, Heinzerling K, et al. Pain therapy guided by purpose and perspective in light of the opioid epidemic. Frontiers in psychiatry. 2018;9.

[8] Hill KP. Medical marijuana for treatment of chronic pain and other medical and psychiatric problems: a clinical review. Jama. 2015;313(24):2474-2483.

[9] Turk DC, Wilson HD, Cahana A. Treatment of chronic non-cancer pain. The Lancet. 2011;377(9784):2226-2235.

[10] Olsen Y, Sharfstein JM. Confronting the stigma of opioid use disorder — and its treatment. Jama. 2014;311(14):13931394.

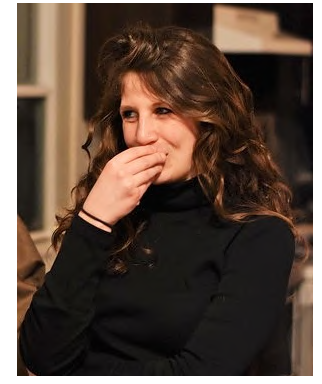

Lola is a PhD student, studying neurosciences at McGill University. Her work focuses on a rodent model of opioid addiction, to study the consequences of long term withdrawal from morphine on the brain and eventually help maintaining abstinence. Never bored talking about brains and minds, she is involved in several science popularization organizations, such as the festival Pint of Science and outreaches to teach some mysteries of brain functioning to teenagers. She is always looking for new occasion to share passions and ideas! 